\title{
Thermal Diffusivity Measurement of Vapor Chamber
}

\author{
Wei-Keng Lin', Wen-Hua Zhang ${ }^{2}$, Pei-Hsun $\mathrm{Wu}^{3}$ \\ ${ }^{1}$ Engineering \& System Science Department, National Tsing-Hua University \\ 101, Sec.2 Kuang Fu Rd., Hsinchu city, Taiwan \\ wklin@ess.nthu.edu.tw \\ 25F., No.143, Zhuangjing 3rd Rd., Zhubei City, Hsinchu, Taiwan (R.O.C.) 30264 \\ vancezhang27@gmail.com \\ ${ }^{3}$ Engineering \& System Science Department, National Tsing-Hua University \\ 101, Sec.2 Kuang Fu Rd., Hsinchu city, Taiwan \\ j6qo4vmp@gmail.com
}

\begin{abstract}
Based on the Angstrom method one dimension theory, this research designed a set of experimental instruments to measure the thermal diffusivity $\alpha$ of the pure material. In addition, the thermal conductivity of the two-phase heat transfer element such as heat pipe and vapor chamber are also be measured. In this experiment, copper, tin, brass, Aluminium alloy has had been measured, the relative error were compared with the data from hand book. This paper also developed a Two-Dimensional theory and had been verified through the experimental data as well.

One dimensional accuracy error are within $10 \%$. The accuracy of the two-dimensional error are at range of 10 to $15 \%$. The experimental results show that the accuracy of the instrument error is less than $10 \%$. The measurement of the heat pipe heat transfer coefficient is always a controversial issue. Using the principle of this paper, the diffusivity of the heat pipe sample can be measured at $213.3\left(\mathrm{~cm}^{2} / \mathrm{s}\right)$, and which is equivalent to the heat transfer coefficient is $25000(\mathrm{~W} / \mathrm{m} . \mathrm{K})$.
\end{abstract}

Keywords: Thermal Diffusivity, Heat Pipe, Vapor Chamber.

\section{Introduction}

With the continuous development of microprocessor performance, the existing CPU volume is getting smaller and smaller, the power density is getting higher and higher. In order to match the good heat transfer of the original, the traditional method is to use the cooling fins [1], but still can't meet the needs of science and technology. In the mobile phone, it is expected that the ultra-thin vapor chamber will have excellent performance in the future [2], the heat transfer principle of the vapor chamber are same as heat pipe, except the direction of heat conduction; In fact, the heat transfer for the vapor chamber is two-dimensional while the heat transfer of heat pipe is one dimension [3].

On the basis of the Angstrom method theory [4], we designed a set of experimental instruments as a measurement instrument. Angstrom method theory is used for pure material analysis, assuming that the vapor chamber is made, its structure is stable, the heat transfer performance is stable as well, and thus the thermal diffusivity of vapopr chamber can be assumed homogeneous, so that Angstrom method is used for the thermal diffusivity measurement on the vapor chamber. The calibration of the test sample using two pure materials and alloys to do the measurement and calculation of instrument error. Pure material selection of copper and tin, the alloy is the choice of Aluminium 6061 and brass [5].

\section{Theory of Thermal Diffusivity Measurement}

In general, thermal diffusivity can be derivate from Angstrom method theory. Once the thermal diffusivity $\alpha$ is obtained, the spread thermal conductivity $K_{s p}$ value can be obtained for $\alpha$ divided by density $\rho$ and heat capacity $C_{p}$. This theory is to heat on the rectangular test sample strip with a periodic cycle. When the sine wave through the conduction to the other end of the strip with a certain constant length and the temperature of the heat wave changes, the use of this temperature amplitude change at both ends of the process will deduce the thermal diffusion coefficient $\alpha$ of the test sample. One-dimensional governor equation can be expressed as Eq. (1). 


$$
\frac{1}{\alpha} \frac{\partial T}{\partial t}=\frac{\partial^{2} T}{\partial x^{2}}-m^{2} T
$$

Where: $m$ is the heat loss of the surface via heat convection

The thermal diffusivity of the object can be calculated by the Eq. (2). The schematic diagram of the measurement is shown in Figure 1. The horizontal axis represents the elapsed time, while the vertical axis represents the temperature scale. The red curve is the temperature at which $\mathrm{T}_{1}$ changes with the heating cycle, $\mathrm{M}$ is the temperature difference between the highest temperature of $T_{1}$ and the lowest temperature, that is, the temperature amplitude of the heating cycle. The blue curve is the temperature at which $\mathrm{T}_{2}$ changes with the heating cycle, $\mathrm{N}$ is the amplitude of $\mathrm{T}_{2}[6]$.

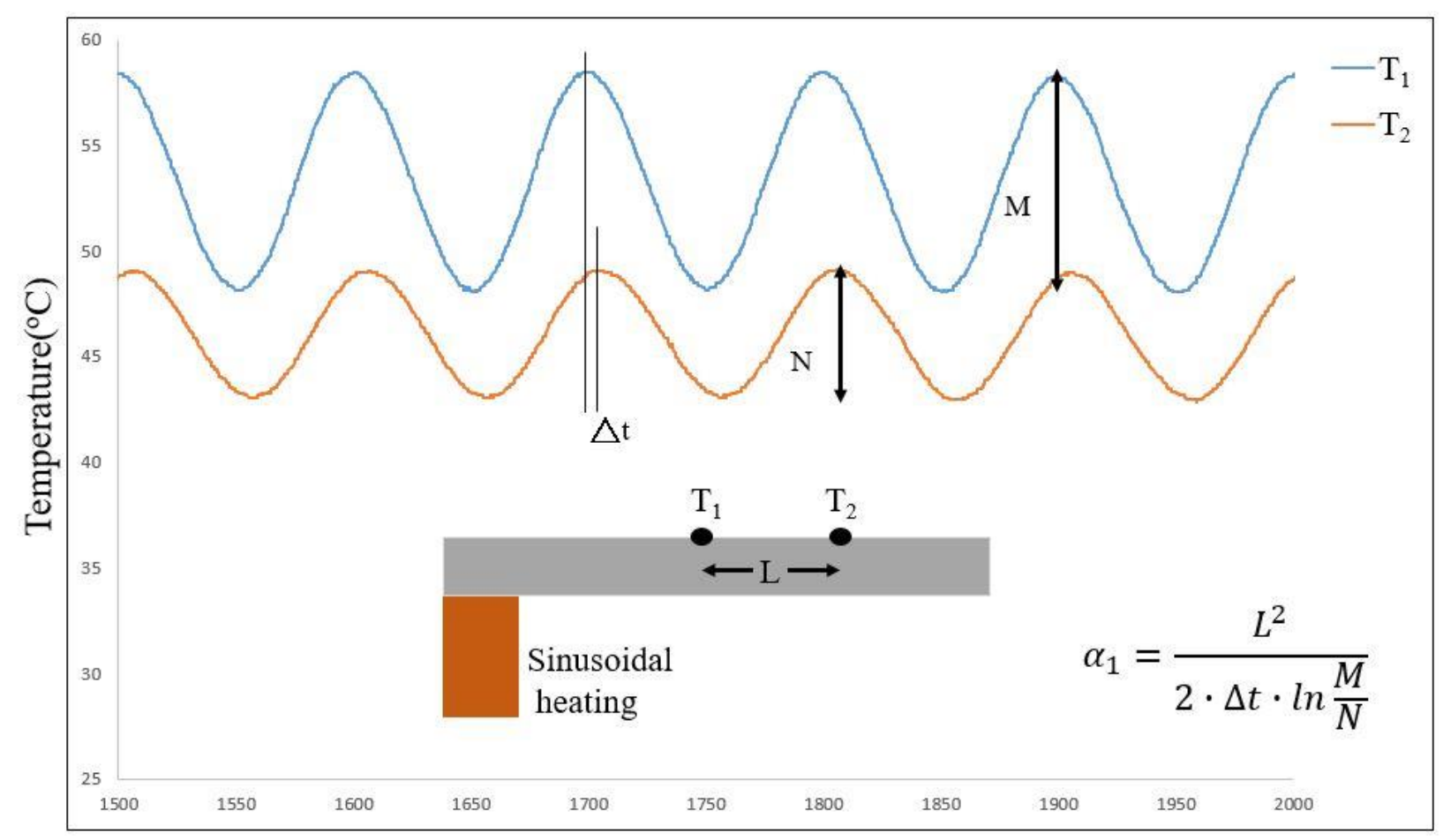

Time(s)

Fig. 1: Schematic diagram of the measurement Angstrom method.

$$
\alpha_{1}=\frac{k}{\rho C_{p}}=\frac{L^{2}}{2 \Delta t\left(\ln \frac{M}{N}\right)}
$$

Where: $\alpha_{1}$ is the one-dimensional thermal diffusivity $\left(\mathrm{cm}^{2} / \mathrm{s}\right), \mathrm{k}$ is the thermal conductivity $(\mathrm{W} / \mathrm{m} . \mathrm{K}), \rho$ for the density $\left(\mathrm{kg} / \mathrm{m}^{3}\right), \mathrm{C}_{\mathrm{p}}$ is the specific heat capacity $(\mathrm{J} / \mathrm{kg} \cdot \mathrm{K}), \mathrm{L}$ is the distance between $\mathrm{T}_{1}$ and $\mathrm{T}_{2}(\mathrm{~cm}), \Delta \mathrm{t}$ is the delay time from $\mathrm{T}_{1}$ to $\mathrm{T}_{2}(\mathrm{~s}), \mathrm{M} / \mathrm{N}$ is the amplitude ratio of $\mathrm{T}_{1}$ and $\mathrm{T}_{2}$.

\section{Experiment}

\subsection{Thermal Diffusivity Measuring Instruments}

Figure 2 shows the heating platform for the thermal diffusivity measurement system, The instrument is used to measure the thermal diffusivity of test planar sample, which comprises a three-seat, two-gauge slide, a and a several of thermocouple temperature probes. Heating fixture arranged in a straight line and contact the surface to be measured to measure the temperature changes of the object, calculate the thermal diffusivity of the test sample. Other devices include power supply, thermocouple connector, NI6009 programmable input controller, RS-232 quick adapter and USB-4718 
thermocouple signal reader etc. Figure 3 shows the power supply, where the power supply provides a sine wave power to achieve the required input power, which is controlled by the NI6009 controller. Through the controller output of the heater control T.E.C. cooling chip to produce the effect of temperature and cooling, a sine wave power is generated through the heat conduction of heating of copper. Heat is transferred to the test sample via the copper block, the thermocouple measurement signal passed to the USB-4718 thermocouple signal reader which treats all the signals data into computerreadable signals to the VB program.

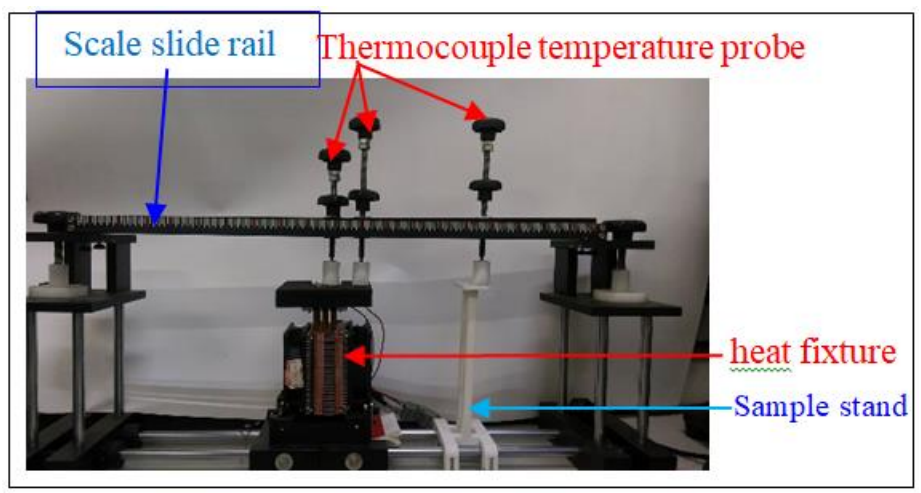

Fig. 2: Heating platform for the thermal diffusivity measurement system.

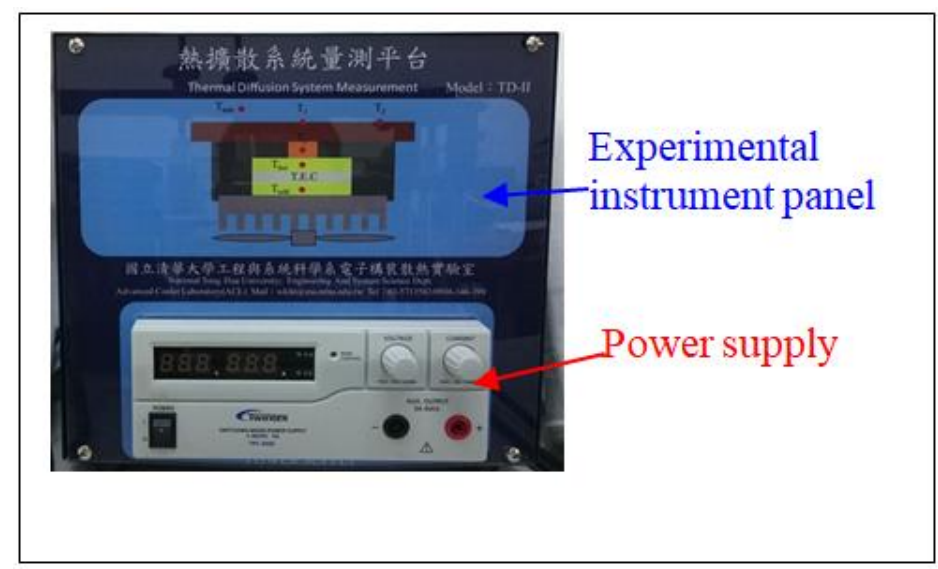

Fig. 3: Sine wave power supply.

\subsection{Experimental Procedure}

In this experiment, the Visual Basic programming language is used to establish the temperature measurement program, and the measurement interface is mainly used to extract the mechanical signal from the data extractor in real time to convert it into actual temperature or actual current, voltage Value, and all the values are updated every 0.1 seconds in the form of the form. In order to verify the accuracy of the data measured by this instrument, we measured some of the known standard values of $\alpha$ standard material to test the error and accuracy. The choice of test pieces on the use of two kinds of pure material and alloy to do comparison. Copper and tin are used for the test of pure material, while Aluminium 6061 and brass are used for the test of alloy. Test sample shown in Figure 4. The aspect ratio of the test piece would be the base criterial used as a one-dimensional analysis and two-dimensional analysis. In order to verify the accuracy of the data measured by this instrument, we measured some of the known standard values of $\alpha$ standard material to test the error and accuracy.

The aspect ratio in the direction of the $\mathrm{X}$ axis is defined by equation (3):

$$
\tau_{\mathrm{x}}=\frac{T}{W}
$$


The aspect ratio in the direction of the $\mathrm{Y}$ axis is defined by equation (4):

$$
\tau_{\mathrm{y}}=\frac{\mathrm{T}}{\mathrm{L}}
$$

Two thermocouple distance is set to $3 \mathrm{~cm}$ as shown in figure 5 , where $T_{1}, T_{2}$ for the temperature measurement points. After the selection is completed, start the program and input the power of the Sine wave change from the power supply shown as in figure 1. Wait about 20 to 30 minutes, until the test piece temperature stable then stop, all the temperature change be recorded and analysis by the program as shown in Figure 6. Hence, the thermal diffusivity $\alpha$ can be calculated by the Angstrom theory method

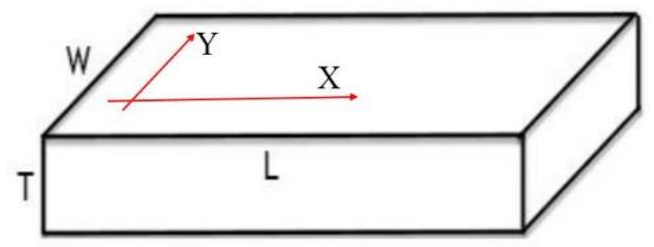

Fig. 4: Schematic diagram of test sample geometric.

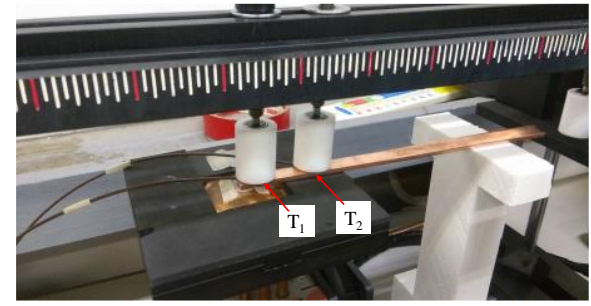

Fig. 5: Test sample strip.

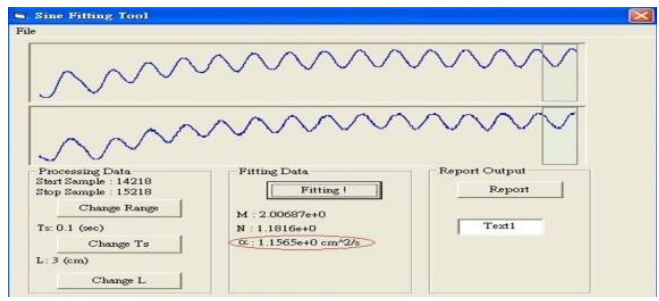

Fig. 6: Program Calculate Thermal Diffusivity $\alpha$ of test sample material.

\section{Results and Discussion}

The data selection portion, in the form of a full-blind test, refers to the measurement and record 3 times in the case of an unknown standard value of $\alpha_{\text {std. }}$. When the repeatability error shown as in Eq. (5) is larger than 10\%, the experiment data will be excluded, thus all the data are classified into stable data. The accuracy error $\varepsilon_{\text {std }}$ shown as in Eq. (6) is to determine the authenticity of the data.

$$
\begin{gathered}
\text { Repeatability error: } \varepsilon_{\text {rep }}=\frac{\left|\alpha_{\text {ave }}-\alpha_{i}\right|}{\alpha_{\text {ave }}} \times 100 \% \\
\text { Accuracy error: } \varepsilon_{\text {std }}=\frac{\left|\alpha_{\text {std }}-\alpha_{i}\right|}{\alpha_{\text {std }}} \times 100 \%
\end{gathered}
$$

where: $\alpha_{1, \text { ave }}=\frac{\sum_{i}^{n} \alpha_{1, i}}{n}, \alpha_{\text {std }}$ is the standard value of the test sample 


\subsection{Thermal Diffusion Rate Analysis of One-dimensional Samples}

Table 1 shows the results of the copper measurement data. It can be seen from Table 1 that the measured $\alpha_{1 \text {,ave }}$ is at range of $1.01 \sim 1.18 \mathrm{~cm}^{2} / \mathrm{s}$, compare with copper standard value $\alpha_{1, \text { std }}=1.17 \mathrm{~cm}^{2} / \mathrm{s}$, the accuracy error of most data are within $10 \%$, only a few are larger than $10 \%$, the largest is $13.68 \%$. Table 2 shows the results of test sample with the same width $\mathrm{W}=10 \mathrm{~mm}$, but different $\mathrm{T}=1 \mathrm{~mm}$ and $\mathrm{T}=2 \mathrm{~mm}$, both $\varepsilon_{1 \text {,std }}$ of two samples are large. The difference between the speculations may be the formation of copper oxide film and surface oil and other impurities. After degrease and pickling treatment, the surface of copper is much brighter. The data is measured again, the results shown as in Table 3 , the $\varepsilon_{1, \text { rep }}$ are within $10 \%$, while $\varepsilon_{1, \text { std }}$ is about $11 \%$. Table 4 shows the test results of tin sample with different thickness and width, it can be seen that $\alpha_{1 \text {,ave }}$ is at range of 0.33 to $0.39 \mathrm{~cm}^{2} / \mathrm{s}$, compare with the $\alpha_{\text {std }}$ of tin $0.40 \mathrm{~cm}^{2} / \mathrm{s}$, the error are within $10 \%$ except two sets of $\mathrm{T}=1 \mathrm{~mm}$, the $\varepsilon_{1, \text { std }}$ is high up to $17.5 \%$, and that could be the effect caused by the oxide layer. The thinner the film, the larger the impact. In this regard, the tin sample were subjected to surface de-oxidation treatment and then measured. The results are shown in Table 5. It can be seen that there are many changes in the two sets of $\mathrm{T}=1 \mathrm{~mm}$ test, the results are much better than the untreated. $\varepsilon_{1, \text { std }}$ have come within $10 \%$. While the test results at $\mathrm{T}=2,3 \mathrm{~mm}$ have also become more accurate. Verifying the metal oxide again will make the measurement error larger. Table 6 is the results for aluminium 6061, It can be seen that each test sample $\alpha_{1, \text { ave }}$ is at range of 0.54 and $0.68 \mathrm{~cm}^{2} / \mathrm{s}$, Compare with the standard value of $\alpha_{\text {std }} 0.62 \mathrm{~cm}^{2} / \mathrm{s}$, the error of $\varepsilon_{1, \text { rep }}$ are within $10 \%$ except at the group of $\mathrm{W}=50, \mathrm{~L}=200, \mathrm{~T}=3$, this group has a greater accuracy error, which up to $17.74 \%$. Table 7 for the brass measurement results, brass for the mixture, the standard value of $\alpha_{\text {std }}$ is $0.34 \mathrm{~cm} 2 / \mathrm{s}$. the $\alpha_{1 \text {,ave }}$ of each test sample is at range of 0.30 to $0.39 \mathrm{~cm}^{2} / \mathrm{s}$, which is close to its standard value, most of the error $\varepsilon_{1 \text {,std }}$ are within $10 \%$. Note that the $10 \times 200 \times 3 \mathrm{~mm}$ test sample, the average of this set of measurements is greater than the other group of brass test sample, presumably because the brass is a mixture, provided by the manufacturers of different brass test samples, its composition may a little bit different. In order to prove this inference, will measure the diffusivity for the same brass sheet before and after cut. If the inference is correct, the measured value of the mother sheet and daughter sheet should be similar. The results are shown in Table 8. Obviously, both the parent brass sheet and the daughter brass sheet $\mathrm{A}$ and $\mathrm{B}$, the measured value is almost the same, and $\varepsilon_{\text {std }}$ are very small, the previous inference is be confirmed: that is from different mother cut out the test sample, the value of the diffusivity are the same and repeatability are similar.

Table 1: Test results of copper sample with different thickness and width $\left(\alpha_{\mathrm{std}}=1.17 \mathrm{~cm}^{2} / \mathrm{s}\right)$.

\begin{tabular}{|c|c|c|c|c|}
\hline Size (mm) & $\alpha_{1}\left(\mathrm{~cm}^{2} / \mathrm{s}\right)$ & $\alpha_{1, \text { ave }}\left(\mathrm{cm}^{2} / \mathrm{s}\right)$ & $\varepsilon_{1, \text { rep }}(\%)$ & $\varepsilon_{1, \text { rep }}(\%)$ \\
\hline \multirow{3}{*}{$\begin{array}{l}\mathrm{W}=50, \quad \mathrm{~L}=200, \\
\mathrm{~T}=1, \tau_{\mathrm{x}}=0.02\end{array}$} & 1.01 & \multirow{3}{*}{1.04} & 3.50 & 13.68 \\
\hline & 1.08 & & 3.18 & 7.69 \\
\hline & 1.05 & & 0.32 & 10.26 \\
\hline \multirow{3}{*}{$\begin{array}{l}\mathrm{W}=50, \quad \mathrm{~L}=200, \\
\mathrm{~T}=2, \tau_{\mathrm{x}}=0.04\end{array}$} & 1.05 & \multirow{3}{*}{1.07} & 2.27 & 10.26 \\
\hline & 1.02 & & 0.65 & 12.82 \\
\hline & 1.01 & & 1.62 & 13.68 \\
\hline \multirow{3}{*}{$\begin{array}{l}\mathrm{W}=50, \quad \mathrm{~L}=200 \\
\mathrm{~T}=3, \tau_{\mathrm{x}}=0.06\end{array}$} & 1.2 & \multirow{3}{*}{1.17} & 2.27 & 2.56 \\
\hline & 1.16 & & 1.14 & 0.85 \\
\hline & 1.16 & & 1.14 & 0.85 \\
\hline \multirow{3}{*}{$\begin{array}{l}\mathrm{W}=30, \quad \mathrm{~L}=200, \\
\mathrm{~T}=1, \tau_{\mathrm{x}}=0.03\end{array}$} & 1.06 & \multirow{3}{*}{1.13} & 6.19 & 9.40 \\
\hline & 1.17 & & 3.54 & 0.00 \\
\hline & 1.16 & & 2.65 & 0.85 \\
\hline \multirow{3}{*}{$\begin{array}{l}\mathrm{W}=30, \quad \mathrm{~L}=200 \\
\mathrm{~T}=2, \tau_{\mathrm{x}}=0.06\end{array}$} & 1.22 & \multirow{3}{*}{1.18} & 3.68 & 4.27 \\
\hline & 1.15 & & 2.27 & 1.71 \\
\hline & 1.16 & & 1.42 & 0.85 \\
\hline \multirow{3}{*}{$\begin{array}{l}\mathrm{W}=30, \quad \mathrm{~L}=200, \\
\mathrm{~T}=3 \quad \tau_{\mathrm{x}}=0.1\end{array}$} & 1.13 & \multirow{3}{*}{1.09} & 3.99 & 3.42 \\
\hline & 1.05 & & 3.37 & 10.26 \\
\hline & 1.08 & & 0.61 & 7.69 \\
\hline \multirow{3}{*}{$\begin{array}{l}\mathrm{W}=9.5, \quad \mathrm{~L}=200 \\
\mathrm{~T}=9.5 \tau_{\mathrm{x}}=1\end{array}$} & 1.17 & \multirow{3}{*}{1.16} & 0.57 & 0.00 \\
\hline & 1.15 & & 1.15 & 1.71 \\
\hline & 1.17 & & 0.57 & 0.00 \\
\hline
\end{tabular}


Table 2: Effect of Oxidation on Copper Test Results $\left(\alpha_{\text {std }}=1.17 \mathrm{~cm}^{2} / \mathrm{s}\right)$.

\begin{tabular}{|c|c|c|c|c|}
\hline Size (mm) & $\begin{array}{c}\alpha_{1} \\
\left(\mathrm{~cm}^{2} / \mathrm{s}\right)\end{array}$ & $\begin{array}{c}\alpha_{1, \text { ave }} \\
\left(\mathrm{cm}^{2} / \mathrm{s}\right)\end{array}$ & $\begin{array}{l}\varepsilon_{1, \text { rep }} \\
(\%)\end{array}$ & $\begin{array}{l}\varepsilon_{1, \text { std }} \\
(\%)\end{array}$ \\
\hline \multirow{3}{*}{$\begin{array}{c}\mathrm{W}=10, \\
\mathrm{~L}=200, \mathrm{~T}=1, \\
\tau_{\mathrm{x}}=0.1\end{array}$} & $\begin{array}{l}0.78 \\
\end{array}$ & \multirow{3}{*}{0.77} & 1.30 & 33.33 \\
\hline & 0.77 & & 0.00 & 34.19 \\
\hline & 0.76 & & 1.30 & 35.04 \\
\hline \multirow{5}{*}{$\begin{array}{c}\mathrm{W}=10, \\
\mathrm{~L}=200, \\
\mathrm{~T}=2, \tau_{\mathrm{x}}=0.2\end{array}$} & 1.2 & \multirow{5}{*}{0.91} & 31.87 & 2.56 \\
\hline & 0.43 & & 52.75 & 63.25 \\
\hline & 0.96 & & 5.49 & 17.95 \\
\hline & 0.84 & & 7.69 & 28.21 \\
\hline & 1.12 & & 23.08 & 4.27 \\
\hline
\end{tabular}

Table 3: Measurement results of copper after treatment $\left(\alpha_{\text {std }}=1.17 \mathrm{~cm}^{2} / \mathrm{s}\right)$.

\begin{tabular}{|c|c|c|c|c|}
\hline Size $(\mathrm{mm})$ & $\begin{array}{c}\alpha_{1} \\
\left(\mathrm{~cm}^{2} / \mathrm{s}\right)\end{array}$ & $\begin{array}{c}\alpha_{1, \text { ave }} \\
\left(\mathrm{cm}^{2} / \mathrm{s}\right)\end{array}$ & $\begin{array}{l}\varepsilon_{1, \text {,ep }} \\
(\%)\end{array}$ & $\begin{array}{r}\varepsilon_{1, \text { std }} \\
(\%)\end{array}$ \\
\hline \multirow{3}{*}{$\begin{array}{c}\mathrm{W}=10 \\
\mathrm{~L}=200, \mathrm{~T}=1 \\
\tau_{\mathrm{x}}=0.1\end{array}$} & 1.02 & \multirow{3}{*}{1.07} & 2.80 & 12.82 \\
\hline & 1.04 & & 4.67 & 11.11 \\
\hline & 1.15 & & 7.48 & 1.71 \\
\hline \multirow{3}{*}{$\begin{array}{c}\mathrm{W}=10, \\
\mathrm{~L}=200, \mathrm{~T}=2, \\
\tau_{\mathrm{x}}=0.2\end{array}$} & 1.08 & \multirow{3}{*}{1.03} & 4.65 & 7.69 \\
\hline & 1.04 & & 0.78 & 11.11 \\
\hline & 1.04 & & 0.78 & 11.11 \\
\hline \multirow{3}{*}{$\begin{array}{c}\mathrm{W}=10, \\
\mathrm{~L}=200, \mathrm{~T}=3, \\
\tau_{\mathrm{x}}=0.3\end{array}$} & 1.13 & \multirow{3}{*}{1.11} & 2.11 & 8.55 \\
\hline & 1.08 & & 2.41 & 7.69 \\
\hline & 1.11 & & 0.30 & 5.13 \\
\hline
\end{tabular}

Table 4: Test results of tin sample with different thickness and width $\left(\alpha_{\mathrm{std}}=0.4 \mathrm{~cm}^{2} / \mathrm{s}\right)$.

\begin{tabular}{|c|c|c|c|c|}
\hline Size $(\mathrm{mm})$ & $\begin{array}{l}\alpha_{1} \\
\left(\mathrm{~cm}^{2} / \mathrm{s}\right)\end{array}$ & $\begin{array}{l}\alpha_{1, \text { ave }} \\
\left(\mathrm{cm}^{2} / \mathrm{s}\right)\end{array}$ & $\begin{array}{l}\varepsilon_{1, \text {,rep }} \\
(\%)\end{array}$ & $\begin{array}{l}\varepsilon_{1, \text { std }} \\
(\%)\end{array}$ \\
\hline \multirow{3}{*}{$\begin{array}{l}\mathrm{W}=20, \quad \mathrm{~L}=230, \\
\mathrm{~T}=1, \tau_{\mathrm{x}}=0.05\end{array}$} & 0.33 & \multirow{3}{*}{0.33} & 1.00 & 17.50 \\
\hline & 0.33 & & 1.00 & 17.50 \\
\hline & 0.34 & & 2.00 & 15.00 \\
\hline \multirow{3}{*}{$\begin{array}{l}\mathrm{W}=20, \quad \mathrm{~L}=230, \\
\mathrm{~T}=2, \tau_{\mathrm{x}}=0.1\end{array}$} & 0.37 & \multirow{3}{*}{0.37} & 0.91 & 7.50 \\
\hline & 0.36 & & 1.82 & 10.00 \\
\hline & 0.37 & & 0.91 & 7.50 \\
\hline \multirow{3}{*}{$\begin{array}{l}\mathrm{W}=20, \quad \mathrm{~L}=230, \\
\mathrm{~T}=3, \tau_{\mathrm{x}}=0.15\end{array}$} & 0.37 & \multirow{3}{*}{0.37} & 4.31 & 7.50 \\
\hline & 0.40 & & 8.11 & 0.00 \\
\hline & 0.39 & & 5.41 & 2.50 \\
\hline \multirow{3}{*}{$\begin{array}{l}\mathrm{W}=30, \quad \mathrm{~L}=230, \\
\mathrm{~T}=1, \tau_{\mathrm{x}}=0.03\end{array}$} & 0.33 & \multirow{3}{*}{0.34} & 1.98 & 17.50 \\
\hline & 0.34 & & 0.99 & 15.00 \\
\hline & 0.34 & & 0.99 & 15.00 \\
\hline \multirow{3}{*}{$\begin{array}{l}\mathrm{W}=30, \quad \mathrm{~L}=230, \\
\mathrm{~T}=2, \tau_{\mathrm{x}}=0.06\end{array}$} & 0.37 & \multirow{3}{*}{0.39} & 5.93 & 7.50 \\
\hline & 0.40 & & 1.69 & 0.00 \\
\hline & 0.41 & & 4.24 & 2.50 \\
\hline \multirow{3}{*}{$\begin{array}{l}\mathrm{W}=30, \quad \mathrm{~L}=230, \\
\mathrm{~T}=3, \tau_{\mathrm{x}}=0.1\end{array}$} & 0.38 & \multirow{3}{*}{0.39} & 1.72 & 5.00 \\
\hline & 0.39 & & 0.86 & 2.50 \\
\hline & 0.39 & & 0.86 & 2.50 \\
\hline
\end{tabular}


Table 5: Measurement results of tin after treatment $\left(\alpha_{\text {std }}=0.4 \mathrm{~cm}^{2} / \mathrm{s}\right)$.

\begin{tabular}{|c|c|c|c|c|}
\hline Size $(\mathrm{mm})$ & $\begin{array}{c}\alpha_{1} \\
\left(\mathrm{~cm}^{2} / \mathrm{s}\right)\end{array}$ & $\begin{array}{c}\alpha_{1, \text { ave }} \\
\left(\mathrm{cm}^{2} / \mathrm{s}\right)\end{array}$ & $\begin{array}{l}\varepsilon_{1, \text { rep }} \\
(\%)\end{array}$ & $\begin{array}{l}\varepsilon_{1, \text { std }} \\
(\%)\end{array}$ \\
\hline \multirow{3}{*}{$\begin{array}{l}\mathrm{W}=20, \\
\mathrm{~L}=230, \quad \mathrm{~T}=1, \\
\tau_{\mathrm{x}}=0.05\end{array}$} & 0.41 & \multirow{3}{*}{0.39} & 6.03 & 2.50 \\
\hline & 0.37 & & 4.31 & 7.50 \\
\hline & 0.38 & & 1.72 & 5.00 \\
\hline \multirow{3}{*}{$\begin{array}{l}\mathrm{W}=20, \\
\mathrm{~L}=230, \quad \mathrm{~T}=2, \\
\tau_{\mathrm{x}}=0.1\end{array}$} & 0.37 & \multirow{3}{*}{0.38} & 2.63 & 7.50 \\
\hline & 0.38 & & 0.00 & 5.00 \\
\hline & 0.39 & & 2.63 & 2.50 \\
\hline \multirow{3}{*}{$\begin{array}{l}\mathrm{W}=20, \\
\mathrm{~L}=230, \quad \mathrm{~T}=3, \\
\tau_{\mathrm{x}}=0.15\end{array}$} & 0.40 & \multirow{3}{*}{0.40} & 0.84 & 0.00 \\
\hline & 0.40 & & 0.84 & 0.00 \\
\hline & 0.39 & & 1.68 & 2.50 \\
\hline \multirow{3}{*}{$\begin{array}{l}\mathrm{W}=30, \mathrm{~L}=230 \\
\mathrm{~T}=1, \tau_{\mathrm{x}}=0.03\end{array}$} & 0.38 & \multirow{3}{*}{0.38} & 0.87 & 5.00 \\
\hline & 0.38 & & 0.87 & 5.00 \\
\hline & 0.39 & & 1.74 & 2.50 \\
\hline \multirow{3}{*}{$\begin{array}{l}\mathrm{W}=30, \\
\mathrm{~L}=230, \quad \mathrm{~T}=2, \\
\tau_{\mathrm{x}}=0.06\end{array}$} & 0.37 & \multirow{3}{*}{0.38} & 1.77 & 7.50 \\
\hline & 0.39 & & 3.54 & 2.50 \\
\hline & 0.37 & & 1.77 & 7.50 \\
\hline \multirow{3}{*}{$\begin{array}{l}\mathrm{W}=30, \\
\mathrm{~L}=230, \quad \mathrm{~T}=3, \\
\tau_{\mathrm{x}}=0.1\end{array}$} & 0.39 & \multirow{3}{*}{0.38} & 2.63 & 2.50 \\
\hline & 0.37 & & 2.63 & 7.50 \\
\hline & 0.38 & & 0.00 & 5.00 \\
\hline
\end{tabular}

Table 6: Test results of Aluminium sample with different thickness and width $\left(\alpha_{\mathrm{std}}=0.4 \mathrm{~cm}^{2} / \mathrm{s}\right)$.

\begin{tabular}{|c|c|c|c|c|}
\hline Size $(\mathrm{mm})$ & $\alpha_{1}\left(\mathrm{~cm}^{2} / \mathrm{s}\right)$ & $\alpha_{1, \text { ave }}\left(\mathrm{cm}^{2} / \mathrm{s}\right)$ & $\varepsilon_{1, \text { rep }}(\%)$ & $\varepsilon_{1, \text { std }}(\%)$ \\
\hline \multirow{3}{*}{$\begin{array}{l}\mathrm{W}=50, \quad \mathrm{~L}=200, \\
\mathrm{~T}=1, \tau_{\mathrm{x}}=0.02\end{array}$} & 0.62 & \multirow{3}{*}{0.62} & 0.00 & 0.00 \\
\hline & 0.60 & & 3.23 & 3.23 \\
\hline & 0.64 & & 3.23 & 3.23 \\
\hline \multirow{3}{*}{$\begin{array}{l}\mathrm{W}=50, \quad \mathrm{~L}=200 \\
\mathrm{~T}=2, \tau_{\mathrm{x}}=0.04\end{array}$} & 0.55 & \multirow{3}{*}{0.58} & 4.62 & 11.29 \\
\hline & 0.56 & & 2.89 & 9.68 \\
\hline & 0.62 & & 7.51 & 0.00 \\
\hline \multirow{3}{*}{$\begin{array}{l}\mathrm{W}=50, \quad \mathrm{~L}=200 \\
\mathrm{~T}=3, \tau_{\mathrm{x}}=0.06\end{array}$} & 0.56 & \multirow{3}{*}{0.54} & 3.70 & 9.68 \\
\hline & 0.51 & & 5.56 & 17.74 \\
\hline & 0.55 & & 1.85 & 11.29 \\
\hline \multirow{3}{*}{$\begin{array}{l}\mathrm{W}=30, \quad \mathrm{~L}=200 \\
\mathrm{~T}=1, \tau_{\mathrm{x}}=0.03\end{array}$} & 0.57 & \multirow{3}{*}{0.55} & 3.64 & 8.06 \\
\hline & 0.53 & & 3.64 & 14.52 \\
\hline & 0.55 & & 0.00 & 11.29 \\
\hline \multirow{3}{*}{$\begin{array}{l}\mathrm{W}=30, \quad \mathrm{~L}=200, \\
\mathrm{~T}=2, \tau_{\mathrm{x}}=0.06\end{array}$} & 0.65 & \multirow{3}{*}{0.67} & 3.47 & 4.84 \\
\hline & 0.69 & & 2.48 & 11.29 \\
\hline & 0.68 & & 0.99 & 9.68 \\
\hline \multirow{3}{*}{$\begin{array}{l}\mathrm{W}=30, \quad \mathrm{~L}=200 \\
\mathrm{~T}=3, \tau_{\mathrm{x}}=0.1\end{array}$} & 0.69 & \multirow{3}{*}{0.68} & 1.97 & 11.29 \\
\hline & 0.69 & & 1.97 & 11.29 \\
\hline & 0.65 & & 3.94 & 4.84 \\
\hline \multirow{3}{*}{$\begin{array}{l}\mathrm{W}=10, \quad \mathrm{~L}=200, \\
\mathrm{~T}=1 \\
\tau_{\mathrm{x}}=0.1\end{array}$} & 0.66 & \multirow{3}{*}{0.65} & 1.02 & 6.45 \\
\hline & 0.62 & & 5.10 & 0.00 \\
\hline & 0.68 & & 4.08 & 9.68 \\
\hline \multirow{3}{*}{$\begin{array}{l}\mathrm{W}=10, \quad \mathrm{~L}=200 \\
\mathrm{~T}=2, \tau_{\mathrm{x}}=0.2\end{array}$} & 0.54 & \multirow{3}{*}{0.55} & 1.22 & 12.90 \\
\hline & 0.53 & & 3.05 & 14.52 \\
\hline & 0.57 & & 4.27 & 8.06 \\
\hline \multirow{3}{*}{$\begin{array}{l}\mathrm{W}=10, \quad \mathrm{~L}=200, \\
\mathrm{~T}=3, \tau_{\mathrm{x}}=0.3\end{array}$} & 0.69 & \multirow{3}{*}{0.66} & 4.55 & 11.29 \\
\hline & 0.67 & & 1.52 & 8.06 \\
\hline & 0.62 & & 6.06 & 0.00 \\
\hline
\end{tabular}


Table 7: Test results of brass sample with different thickness and width $\left(\alpha_{\mathrm{std}}=0.34 \mathrm{~cm}^{2} / \mathrm{s}\right)$.

\begin{tabular}{|c|c|c|c|c|}
\hline Size $(\mathrm{mm})$ & $\alpha_{1}\left(\mathrm{~cm}^{2} / \mathrm{s}\right)$ & $\alpha_{1, \text { ave }}\left(\mathrm{cm}^{2} / \mathrm{s}\right)$ & $\varepsilon_{1, \text { rep }}(\%)$ & $\varepsilon_{1, \text { std }}(\%)$ \\
\hline \multirow{3}{*}{$\begin{array}{l}\mathrm{W}=50, \quad \mathrm{~L}=200, \\
\mathrm{~T}=1, \tau_{\mathrm{x}}=0.02\end{array}$} & 0.32 & \multirow{3}{*}{0.33} & 2.04 & 5.88 \\
\hline & 0.35 & & 7.14 & 2.94 \\
\hline & 0.31 & & 5.10 & 8.82 \\
\hline \multirow{3}{*}{$\begin{array}{l}\mathrm{W}=50, \quad \mathrm{~L}=200, \\
\mathrm{~T}=2, \tau_{\mathrm{x}}=0.04\end{array}$} & 0.30 & \multirow{3}{*}{0.31} & 3.23 & 11.76 \\
\hline & 0.32 & & 3.23 & 5.88 \\
\hline & 0.31 & & 0.00 & 8.82 \\
\hline \multirow{3}{*}{$\begin{array}{l}\mathrm{W}=50, \quad \mathrm{~L}=200, \\
\mathrm{~T}=3, \tau_{\mathrm{x}}=0.06\end{array}$} & 0.29 & \multirow{3}{*}{0.30} & 3.33 & 14.71 \\
\hline & 0.31 & & 3.33 & 8.82 \\
\hline & 0.30 & & 0.00 & 11.76 \\
\hline \multirow{3}{*}{$\begin{array}{l}\mathrm{W}=30, \quad \mathrm{~L}=200, \\
\mathrm{~T}=1, \tau_{\mathrm{x}}=0.03\end{array}$} & 0.31 & \multirow{3}{*}{0.31} & 1.09 & 8.82 \\
\hline & 0.31 & & 1.09 & 8.82 \\
\hline & 0.30 & & 2.17 & 11.76 \\
\hline \multirow{3}{*}{$\begin{array}{l}\mathrm{W}=30, \quad \mathrm{~L}=200, \\
\mathrm{~T}=2, \tau_{\mathrm{x}}=0.06\end{array}$} & 0.31 & \multirow{3}{*}{0.32} & 3.13 & 8.82 \\
\hline & 0.33 & & 3.13 & 2.94 \\
\hline & 0.32 & & 0.00 & $\begin{array}{l}5.88 \\
\end{array}$ \\
\hline \multirow{3}{*}{$\begin{array}{l}\mathrm{W}=30, \quad \mathrm{~L}=200, \\
\mathrm{~T}=3, \tau_{\mathrm{x}}=0.1\end{array}$} & 0.33 & \multirow{3}{*}{0.33} & 1.02 & 2.94 \\
\hline & 0.32 & & 2.04 & 5.88 \\
\hline & 0.33 & & 1.02 & 2.94 \\
\hline \multirow{3}{*}{$\begin{array}{l}\mathrm{W}=10, \quad \mathrm{~L}=200, \\
\mathrm{~T}=1, \tau_{\mathrm{x}}=0.1\end{array}$} & 0.36 & \multirow{3}{*}{0.35} & 2.86 & $\begin{array}{l}5.88 \\
\end{array}$ \\
\hline & 0.36 & & 286 & 5.88 \\
\hline & 0.33 & & 5.71 & 2.94 \\
\hline \multirow{3}{*}{$\begin{array}{l}\mathrm{W}=10, \quad \mathrm{~L}=200, \\
\mathrm{~T}=2, \tau_{\mathrm{x}}=0.2\end{array}$} & 0.32 & \multirow{3}{*}{0.34} & 5.88 & 5.88 \\
\hline & 0.34 & & 0.00 & 0.00 \\
\hline & 0.36 & & $\begin{array}{l}5.88 \\
\end{array}$ & 5.88 \\
\hline \multirow{3}{*}{$\begin{array}{l}\mathrm{W}=10, \quad \mathrm{~L}=200, \\
\mathrm{~T}=3, \tau_{\mathrm{x}}=0.3\end{array}$} & 0.39 & \multirow{3}{*}{0.39} & 0.00 & 14.71 \\
\hline & 0.39 & & 0.00 & 14.71 \\
\hline & 0.39 & & 0.00 & 14.71 \\
\hline \multirow{3}{*}{$\begin{array}{l}\mathrm{W}=9.5, \quad \mathrm{~L}=200, \\
\mathrm{~T}=9.5, \tau_{\mathrm{x}}=1\end{array}$} & 0.35 & \multirow{3}{*}{0.34} & 3.96 & 2.94 \\
\hline & 0.34 & & 0.99 & 0.00 \\
\hline & 0.32 & & 4.95 & 5.88 \\
\hline
\end{tabular}

Table 8: Test results before and after brass cutting $\left(\alpha_{\mathrm{std}}=0.34 \mathrm{~cm}^{2} / \mathrm{s}\right)$.

\begin{tabular}{|c|c|c|c|c|}
\hline Size $(\mathrm{mm})$ & $\alpha_{1}\left(\mathrm{~cm}^{2} / \mathrm{s}\right)$ & $\alpha_{1, \text { ave }}\left(\mathrm{cm}^{2} / \mathrm{s}\right)$ & $\varepsilon_{1, \text { rep }}(\%)$ & $\varepsilon_{1, \text { std }}(\%)$ \\
\hline \multirow{3}{*}{$\begin{array}{l}\mathrm{W}=30, \mathrm{~L}=200, \mathrm{~T}=3, \\
\tau_{\mathrm{x}}=0.1, \text { Mother sheet }\end{array}$} & 0.34 & \multirow{3}{*}{0.34} & 0.99 & 0.00 \\
\hline & 0.34 & & 0.99 & 0.00 \\
\hline & 0.33 & & 1.98 & 2.94 \\
\hline \multirow{3}{*}{$\begin{array}{l}\mathrm{W}=15, \mathrm{~L}=200, \mathrm{~T}=3, \\
\tau_{\mathrm{x}}=0.2, \text { Daughter sheet } \\
\text { A }\end{array}$} & 0.34 & \multirow{3}{*}{0.33} & 4.08 & 0.00 \\
\hline & 0.32 & & 2.04 & 5.88 \\
\hline & 0.32 & & 2.04 & 5.88 \\
\hline \multirow{3}{*}{$\begin{array}{l}\mathrm{W}=10, \mathrm{~L}=200, \mathrm{~T}=3, \\
\tau_{\mathrm{x}}=0.3, \text { Daughter sheet } \\
\mathrm{B}\end{array}$} & 0.34 & \multirow{3}{*}{0.34} & \multirow{3}{*}{0.99} & \multirow{3}{*}{2.94} \\
\hline & 0.34 & & & \\
\hline & 0.33 & & & \\
\hline
\end{tabular}

\subsection{Analysis of Thermal Diffusivity of Two-dimensional Samples}

For the two-dimensional analysis, the sample are copper and brass with 90x90mm and 120x120mm two sizes respectively. Calculated through equation (2) and compared to its repeatability 、 accuracy, and corrected to get $\alpha_{2}$ 。Table 9 is for the measurement results of copper, the $\alpha 1$, ave is at range of 0.52 to $0.65 \mathrm{~cm}^{2} / \mathrm{s}$, although there is a good repeatability, but the error of $\varepsilon_{1, \text { std }}$ is large, the error is range from $43.59 \%$ to $56.84 \%$. Table 10 is for the measurement 
results of brass, the $\alpha_{1, \text { ave }}$ is at range of 0.16 to $0.17 \mathrm{~cm}^{2} / \mathrm{s}$, the same good repeatability, but the error of $\varepsilon_{1, \text { std }}$ is large, the error is range from $50 \%$ to $52.94 \%$ the accuracy is not good enough.

Comparing the results of these two test samples, assuming that the test samples are homogeneous, if the thermal diffusivity measured in one dimension is multiplied by 2 that is $\alpha_{2}=2 \alpha_{1}$, where $\alpha_{1}$ is the calculated data based on one dimension theory, while $\alpha_{2}$ is the calculated data based on two-dimension theory. The results was shown in Table 9. Get $\alpha_{2, \text { ave }}$ is at range from 1.03 to $1.30 \mathrm{~cm}^{2} / \mathrm{s}$, and that become close to $\alpha_{\text {std }}$ and $\varepsilon_{2, \text { std }}$ is around $10 \%$, the largest is $13.68 \%$. Table 4-10 is the test results for brass sample, the calculated diffusivity data $\alpha_{2, \text { ave }}$ based on two dimension theory is at range from 0.32 to $0.34 \mathrm{~cm}^{2} / \mathrm{s}$, and that become close to the $\alpha_{\text {std }}$, all the error of $\varepsilon_{2, \text { std }}$ are less than $10 \%$. From the results shown in Table 9 and Table 10, the assumed $\alpha_{2}=2 \alpha_{1}$ can be used in the measurement of two-dimensional test sample. For the case of $\tau_{\mathrm{x}} \geqq$ $0.02\left(\mathrm{~W}=50 \mathrm{~mm}, \mathrm{~T}=1 \mathrm{~mm}, \tau_{\mathrm{x}}=0.02\right)$, one-dimensional analysis can be applied; while for the case of $\tau_{\mathrm{x}}<0.02(\mathrm{~W}=$ $\left.90 \mathrm{~mm}, \mathrm{~T}=1 \mathrm{~mm}, \tau_{\mathrm{x}}=0.011\right)$, two-dimensional analysis is used.

Table 9: Test results of copper sample with different thickness and width $\left(\alpha_{\mathrm{std}}=1.17 \mathrm{~cm}^{2} / \mathrm{s}\right)$.

\begin{tabular}{|c|c|c|c|c|c|c|c|}
\hline Size $(\mathrm{mm})$ & $\alpha_{1}\left(\mathrm{~cm}^{2} / \mathrm{s}\right)$ & $\alpha_{1, \text { ave }}\left(\mathrm{cm}^{2} / \mathrm{s}\right)$ & $\varepsilon_{1, \text { rep }}(\%)$ & $\varepsilon 1$, std $(\%)$ & $\alpha_{2}=2 \alpha_{1}\left(\mathrm{~cm}^{2} / \mathrm{s}\right)$ & $\alpha_{2, \text { ave }}\left(\mathrm{cm}^{2} / \mathrm{s}\right)$ & $\varepsilon 2$,std $(\%)$ \\
\hline \multirow{3}{*}{$\begin{array}{l}\mathrm{W}=90, \\
\mathrm{~L}=90, \mathrm{~T}=1, \\
\tau_{\mathrm{x}}=0.011\end{array}$} & 0.66 & \multirow{3}{*}{0.65} & 1.02 & 43.59 & 1.32 & \multirow{3}{*}{1.30} & 12.82 \\
\hline & 0.64 & & 2.04 & 45.30 & 1.28 & & 9.40 \\
\hline & 0.66 & & 1.02 & 43.59 & 1.32 & & 12.82 \\
\hline \multirow{2}{*}{$\begin{array}{l}\mathrm{W}=90, \\
\mathrm{~L}=90, \mathrm{~T}=0.6, \\
\tau_{\mathrm{x}}=0.006\end{array}$} & 0.65 & \multirow[t]{2}{*}{0.65} & 0.00 & 44.44 & 1.30 & \multirow[t]{2}{*}{1.30} & 11.11 \\
\hline & 0.65 & & 0.00 & 44.44 & 1.30 & & 11.11 \\
\hline $\begin{array}{l}\mathrm{W}=90, \\
\mathrm{~L}=90, \mathrm{~T}=0.4, \\
\tau_{\mathrm{x}}=0.004\end{array}$ & 0.61 & 0.58 & 4.57 & 47.86 & 1.22 & 1.17 & 4.27 \\
\hline \multirow{3}{*}{$\begin{array}{l}\mathrm{W}=120, \\
\mathrm{~L}=120, \mathrm{~T}=1, \\
\tau_{\mathrm{x}}=0.008\end{array}$} & 0.52 & \multirow{3}{*}{0.52} & 0.65 & 55.73 & 1.04 & \multirow{3}{*}{1.03} & 11.45 \\
\hline & 0.52 & & 0.65 & 55.47 & 1.04 & & 10.94 \\
\hline & 0.51 & & 1.29 & 56.84 & 1.02 & & 13.68 \\
\hline \multirow{2}{*}{$\begin{array}{l}\mathrm{W}=120, \\
\mathrm{~L}=120, \mathrm{~T}=0.6, \\
\tau_{\mathrm{x}}=0.005\end{array}$} & 0.59 & \multirow[b]{2}{*}{0.60} & 1.12 & 49.57 & 1.18 & \multirow[b]{2}{*}{1.19} & 0.85 \\
\hline & 0.60 & & 0.56 & 48.72 & 1.20 & & 2.56 \\
\hline
\end{tabular}

Table 10: Test results of brass sample with different thickness and width $\left(\alpha_{\mathrm{std}}=0.34 \mathrm{~cm}^{2} / \mathrm{s}\right)$.

\begin{tabular}{|c|c|c|c|c|c|c|c|}
\hline Size (mm) & $\alpha_{1}\left(\mathrm{~cm}^{2} / \mathrm{s}\right)$ & $\alpha_{1, \text { ave }}\left(\mathrm{cm}^{2} / \mathrm{s}\right)$ & $\varepsilon_{1, \text { rep }}(\%)$ & $\varepsilon_{1, \text { std }}(\%)$ & $\alpha_{2}=2 \alpha_{1}\left(\mathrm{~cm}^{2} / \mathrm{s}\right)$ & $\alpha_{2, \text { ave }}\left(\mathrm{cm}^{2} / \mathrm{s}\right)$ & $\varepsilon_{2, \text { std }}(\%)$ \\
\hline \multirow{3}{*}{$\begin{array}{l}\mathrm{W}=90, \quad \mathrm{~L}=90 \\
\mathrm{~T}=1, \tau_{\mathrm{x}}=0.011\end{array}$} & 0.16 & \multirow{3}{*}{0.17} & 4.00 & 52.94 & 0.32 & \multirow{3}{*}{0.33} & 5.88 \\
\hline & 0.17 & & 2.00 & 50.00 & 0.34 & & 0.00 \\
\hline & 0.17 & & 2.00 & 50.00 & 0.34 & & 0.00 \\
\hline \multirow{2}{*}{$\begin{array}{l}\mathrm{W}=90, \quad \mathrm{~L}=90 \\
\mathrm{~T}=0.6, \tau_{\mathrm{x}}=0.006\end{array}$} & 0.16 & \multirow[t]{2}{*}{0.16} & 0.00 & 52.94 & 0.32 & \multirow[t]{2}{*}{0.32} & 5.88 \\
\hline & 0.16 & & 0.00 & 52.94 & 0.32 & & 5.88 \\
\hline \multirow{2}{*}{$\begin{array}{l}\mathrm{W}=90, \quad \mathrm{~L}=90, \\
\mathrm{~T}=0.4, \tau_{\mathrm{x}}=0.004\end{array}$} & 0.16 & \multirow{2}{*}{0.16} & 0.00 & 52.94 & 0.32 & \multirow{2}{*}{0.32} & 5.88 \\
\hline & 0.16 & & 0.00 & 52.94 & 0.32 & & 5.88 \\
\hline \multirow{3}{*}{$\begin{array}{l}\mathrm{W}=120, \mathrm{~L}=120 \\
\mathrm{~T}=1, \tau_{\mathrm{x}}=0.008\end{array}$} & 0.16 & \multirow{3}{*}{0.17} & 2.00 & 52.06 & 0.33 & \multirow{3}{*}{0.34} & 2.94 \\
\hline & 0.17 & & 3.41 & 49.41 & 0.34 & & 0.00 \\
\hline & 0.16 & & 1.40 & 51.76 & 0.33 & & 2.94 \\
\hline \multirow{2}{*}{$\begin{array}{l}\mathrm{W}=120, \mathrm{~L}=120 \\
\mathrm{~T}=0.6, \tau_{\mathrm{x}}=0.005\end{array}$} & 0.17 & \multirow[b]{2}{*}{0.17} & 0.00 & 50.00 & 0.34 & \multirow[b]{2}{*}{0.34} & 0.00 \\
\hline & 0.17 & & 0.00 & 50.00 & 0.34 & & 0.00 \\
\hline
\end{tabular}




\subsection{Analysis of Measurement and Application of Thermal Conductivity of Heat Pipe and Vapor Chamber}

Select a heat pipe sample as shown in Figure 7 , diameter $0.85 \mathrm{~cm}$ length $16.7 \mathrm{~cm}$. since the aspect ratio thickness to widthness $\tau_{\mathrm{x}}=1$ which is larger than 0.02 , therefore use one-dimensional analysis. The results of the measurement by Angstrom are shown in Table 11 , and $\alpha_{1, \text { ave }}=213.3 \mathrm{~cm}^{2} / \mathrm{s}$ is measured. The actual density of $1.75 \mathrm{~g} / \mathrm{cm}^{3}$, and heat capacity of $0.68 \mathrm{~J} / \mathrm{g} .{ }^{\circ} \mathrm{C}$, plug all these data into the Eq. (2) get the thermal conductivity of the heat pipe sample $\mathrm{k}$ value of 25000 (W/m.K). Vapor chamber as shown in Figure 8, the size of $90 \mathrm{x} 90 \mathrm{x} 1.5 \mathrm{~mm}, \tau_{\mathrm{x}}=\tau_{\mathrm{y}}=0.016$, are less than 0.02 , according to the previous speculation, should use two-dimensional analysis to do the calculation, the calculation results shown in Table 12. It can be seen that the thermal diffusivity of this particular vapor chamber is about three times that of copper. It is important to note that there are some thermal diffusivity differences between the $\mathrm{X}$ direction and the $\mathrm{Y}$ direction and could be due to the effect of the capillary structure different orientation in vapor chamber.

Table 11: Test results of heat pipe sample.

\begin{tabular}{|l|c|c|}
\hline \multicolumn{1}{|c|}{ Size $(\mathrm{cm})$} & $\alpha_{1}\left(\mathrm{~cm}^{2} / \mathrm{s}\right)$ & $\alpha_{1, \text { ave }}\left(\mathrm{cm}^{2} / \mathrm{s}\right)$ \\
\hline $\begin{array}{l}\phi=0.85, \\
\mathrm{~L}=16.7,\end{array}$ & 230 & \multirow{2}{*}{213.3} \\
\cline { 2 - 2 }$\tau_{\mathrm{x}}=1$ & 210 & \multirow{2}{*}{213.3} \\
\cline { 2 - 2 } & 200 & \\
\hline
\end{tabular}

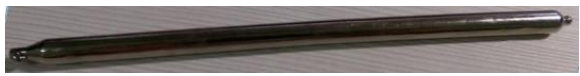

Fig. 7: Heat pipe sample.

Table 12: Test results of vapour chamber sample.

\begin{tabular}{|c|c|c|c|}
\hline $\operatorname{Size}(\mathrm{mm})$ & $\alpha_{2}\left(\mathrm{~cm}^{2} / \mathrm{s}\right)$ & $\alpha_{2, \text { ave }}\left(\mathrm{cm}^{2} / \mathrm{s}\right)$ & $\varepsilon_{2, \text { rep }}(\%)$ \\
\hline \multirow{3}{*}{$\begin{array}{l}\mathrm{W}=90, \quad \mathrm{~L}=90, \\
\mathrm{~T}=2, \\
\tau_{\mathrm{x}}=0.016, \quad \mathrm{X}- \\
\text { direction }\end{array}$} & 3.40 & \multirow{3}{*}{3.24} & 4.94 \\
\hline & 3.04 & & 6.17 \\
\hline & 3.28 & & 1.23 \\
\hline \multirow{3}{*}{$\begin{array}{l}\mathrm{W}=90, \quad \mathrm{~L}=90, \\
\mathrm{~T}=2, \\
\tau_{\mathrm{y}}=0.016, \quad \mathrm{Y}- \\
\text { direction }\end{array}$} & 3.60 & \multirow{3}{*}{3.65} & 1.28 \\
\hline & 3.74 & & 2.56 \\
\hline & 3.60 & & 1.28 \\
\hline
\end{tabular}

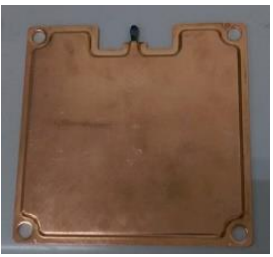

Fig. 8: vapor chamber sample.

\section{Conclusion}

1. The instrument in the measurement of the thermal diffusivity have high repeatability and high accuracy which consistent with the "Angstorm theory".

2. The $\alpha_{1 \text {,ave }}$ of the one-dimensional copper test sample is $1.01 \sim 1.18 \mathrm{~cm}^{2} / \mathrm{s}$, compare with the standard value of $\alpha_{\text {std }}=$ $1.17 \mathrm{~cm}^{2} / \mathrm{s}$, the $\varepsilon_{1, \mathrm{std}}$ is $13 \%$. The results show that $\alpha_{1, \text { ave }}$ of Tin is $0.38 \sim 0.40 \mathrm{~cm}^{2} / \mathrm{s}$ after surface treatment, and that is close to $\alpha_{\text {std }}=0.40 \mathrm{~cm}^{2} / \mathrm{s}$. The $\alpha_{1, \text { ave }}$ of the aluminium 6061 test sample is $0.54 \sim 0.68 \mathrm{~cm}^{2} / \mathrm{s}$, compare with the $\varepsilon_{1, \text { std }}$ of $\alpha_{\text {std }} 0.62$ $\mathrm{cm}^{2} / \mathrm{s}$, the error is $12 \%$. The brass test sample $\alpha_{1, \text { ave }}$ is $0.30 \sim 0.39 \mathrm{~cm}^{2} / \mathrm{s}$, compare with the $\varepsilon_{1, \mathrm{std}}$ of $\alpha_{\text {std }} 0.34 \mathrm{~cm}^{2} / \mathrm{s}$, the error 
is at most $14 \%$.

3. Oxidation and surface oil pollution on the metal is quite significant on the measurement of $\alpha$, that will make the measured value of $\alpha$ decreased large, especially for the high $\alpha$ materials such as copper. Hence the pre-treatment processing is very important.

4. In this experiment, $\alpha_{2}=2 \alpha_{1}$ describe the relation between $\alpha_{2}$ and $\alpha_{1}$, and that has been verified by the test of copper and brass sample with size of $90 \times 90 \mathrm{~mm}$ and $120 \times 120 \mathrm{~mm}$ respectively. The error $\varepsilon_{2, \text { std }}$ of copper is about $10 \%$ and error of brass $\varepsilon_{2, \text { std }}$ are less than $10 \%$. Judgment of one-dimensional or two-dimensional analysis of the material is based on the thickness ratio $\tau_{\mathrm{x}}$ and $\tau_{\mathrm{y}}$. When $\tau<0.02$, use two-dimensional formula. When $\tau \geqq 0.02$, use one-dimensional formula.

5. The thermal conductivity of the heat pipe sample was equivalent to $25000(\mathrm{~W} / \mathrm{m} . \mathrm{K})$ in this experiment, and the same manners can be applied to vapor chamber.

\section{References}

[1] J. E. Parrott and A. D. Stuckes, "Thermal Conductivity of Solids," Pion Limited, London, 1975.

[2] S.-C. Wong K,-C. Hsieh, J.-D. Wu, Wu.-L. Han, "Experiments on a novel vapor chamber," ITHERM 2008 Conference, Orlando, FL, USA, 2008.

[3] Y. Hanzhou, "Study on Feasibility of Measuring Vapor Chamber by Angstorm Method," M.S. thesis, Engineering \& System Science Department, National Tsing-Hua University, Taiwan, 2013.

[4] A. M. Bouchard, "Angstrom's Method of Determining Thermal Conductivity," Physics Department, The College of Wooster, Ohio 44691, 2000.

[5] Z. Goods Yan, "Study on the Influence of Measurement Parameters of Vapor Chamber," M.S. thesis, Engineering \& System Science Department, National Tsing-Hua University, Taiwan, 2014.

[6] Chen Zhekai, "Study on the Establishment of Standard Test Experiment of Vapor Chamber and its Influence of Parameters,” M.S. thesis, Engineering \& System Science Department, National Tsing-Hua University, Taiwan, 2016. 